\title{
The mechanism of the light variability of chemically peculiar stars
}

\author{
J. Krtička ${ }^{1}$, Z. Mikulášek ${ }^{1}$, J. Zverko ${ }^{2}$, J. Žižňovský ${ }^{2}$, G. W. Henry ${ }^{3}$, \\ J. Skalický ${ }^{1}$ and P. Zvěřina ${ }^{1}$ \\ ${ }^{1}$ Institute of Theoretical Physics and Astrophysics, Masaryk University, CZ-611 37 Brno, \\ Czech Republic \\ ${ }^{2}$ Astronomical Institute, Slovak Academy of Sciences, SK-059 60 Tatranská Lomnica, Slovakia \\ ${ }^{3}$ Center of Excellence in Information Systems, Tennessee State University, Nashville, \\ Tennessee, USA
}

\begin{abstract}
Until recently, the mechanism of the light variability of chemically peculiar (CP) stars was unclear. To improve this situation, we started a theoretical and observational campaign aimed at the nature of the light variability of these stars. We use the TLUSTY model atmospheres calculated for the appropriate surface chemical composition to obtain the emergent flux and to predict the rotationally modulated light curves. We show on example of several wellstudied CP stars that their light variations can be explained as a result of i) the uneven surface distribution of the elements (creating overabundant regions), ii) the flux redistribution from the ultraviolet to the visible part of the spectrum (in the overabundant regions), and iii) rotation of the star. We show that the silicon and helium bound-free transitions and iron bound-bound transitions provide the main contribution to the flux redistribution. This result is also a very precise test of modern stellar model atmospheres. We conclude that the mentioned mechanism is a very promising explanation for the light variations in CP stars of earlier spectral types.
\end{abstract}

Keywords. stars: atmospheres, stars: chemically peculiar, stars: spots, stars: variables: other

\section{Magnetic chemically peculiar stars}

Magnetic chemically peculiar stars belong to one of a few groups of variable stars whose light variations are not very well understood. These stars show inhomogeneous surface distribution of different elements, including helium, silicon or iron (see Fig. 1). Krtička et al. (2007), and Krtička et al. (2009) showed that the redistribution of the emergent flux due to the bound-free (continuum) and bound-bound (line) transitions in the stellar atmosphere (Fig. 3) is a mechanism explaining the observed light variations (Fig. 2). Moreover, the comparison of observed and simulated light variations can provide an important new test of modern model stellar atmospheres.

\section{Light curve calculation}

The models of the stellar atmospheres are calculated using the code TLUSTY (Hubeny \& Lanz 1995, Lanz \& Hubeny 2007) assuming a fixed stellar effective temperature and surface gravity. The abundance of chemical elements concerned is set in agreement with the maps of surface elemental distribution derived from spectroscopy (Fig. 1).

The emergent fluxes from individual surface elements are calculated using the code SYNSPEC. From these fluxes the magnitudes of the star in individual filters of Strömgren photometric system are derived (Fig. 3). Finally, these simulated light variations are compared with the observed ones (Fig. 2). We do not use any free parameter to fit the observed light curves. 

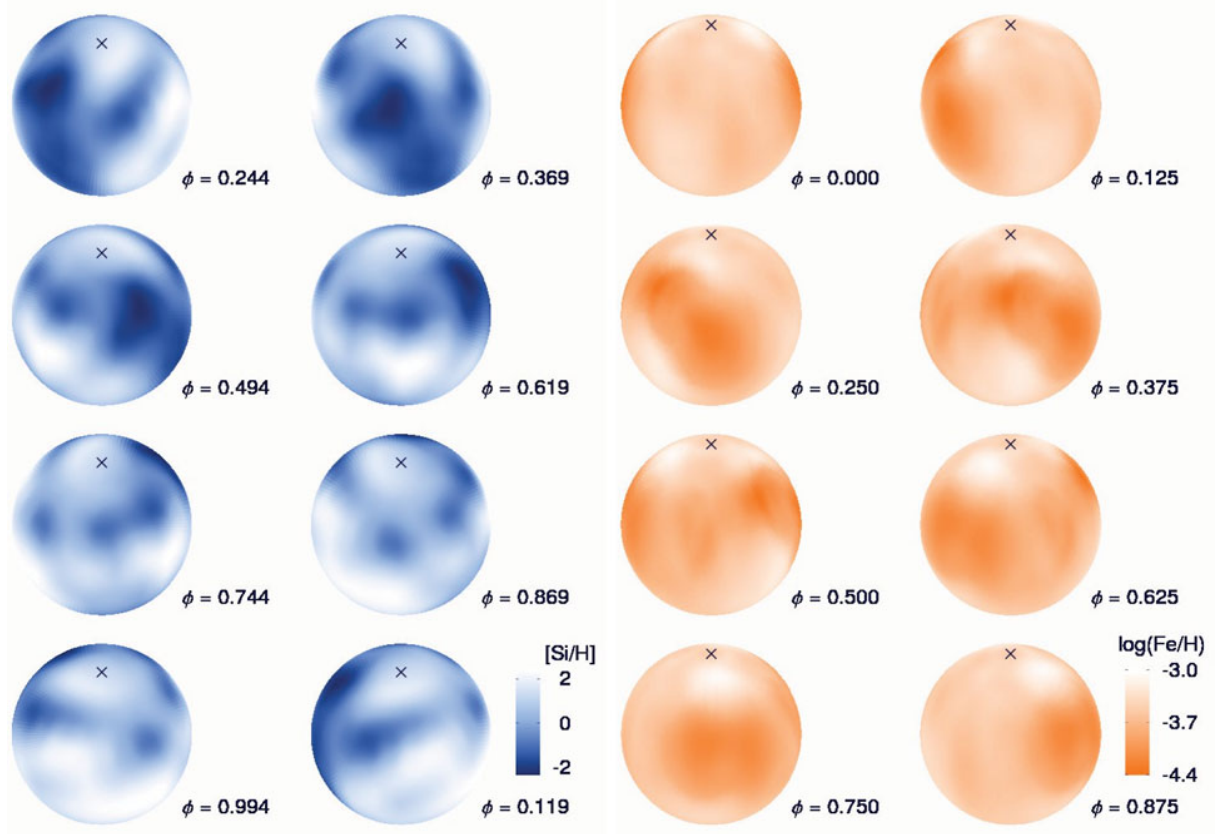

Figure 1. Left: The variation of silicon abundances on the visible disc of HD 37776 for different rotational phases after Khokhlova et al. (2000). Silicon is overabundant on the visible disc around the phase $\phi=0$. Right: The variation of iron abundances on the visible disc of HR 7224 for different rotational phases after Lehmann et al. (2007). Iron is overabundant on the visible disc around the phase $\phi=0$.

Here we use the ephemeris derived by Mikulášek et al. (2008) for HD 37776 and by Krtička et al. (2009) for HR 7224.

\section{Conclusions}

We conclude that the observed light variations of HR 7224 and HD 37776 can be explained as a result of inhomogeneous distribution of helium, silicon and iron on the surface of these stars. This causes the redistribution of the flux from the ultraviolet to the visible part of the spectrum, and, due to the stellar rotation, the light variations. Our models are also able to reproduce the $\mathrm{H} \beta$ line variability of $\mathrm{HR} 7224$.

A very good agreement of the observed and simulated light variations serves as an independent test of modern model stellar atmospheres. The successful comparison of the predicted and observed light curves would not be possible without using the calculations of radiative ionization cross-section done by the Opacity Project team (Seaton et al. 1992).

\section{Acknowledgement}

This work was supported by grant GAAV IAA301630901 and VEGA 2/0074/09.

\section{References}

Adelman, S. J. 1997a, A $\mathscr{B} A S, 122,249$

Adelman, S. J. 1997b, AEBAS, 125, 65

Adelman, S. J. \& Pyper, D. M. 1985, A\& AS, 62, 279 

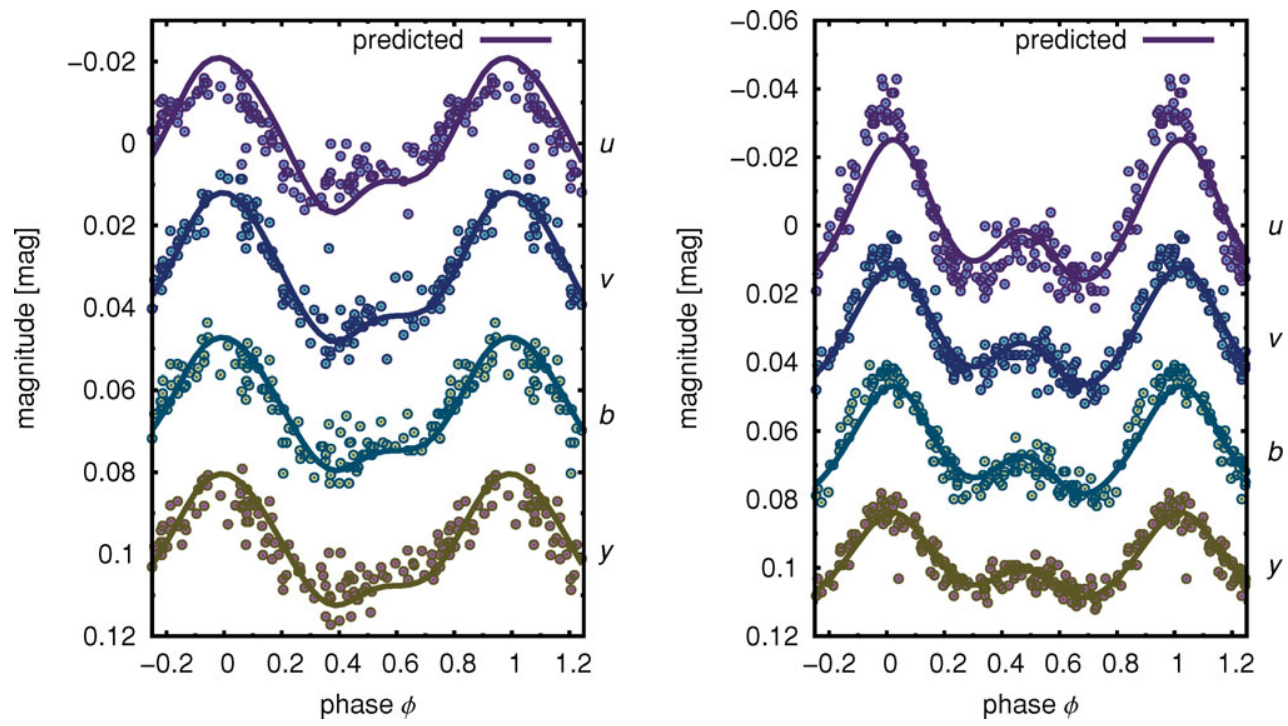

Figure 2. Left: Comparison of the predicted light variations of HD 37776 (calculated using the silicon and helium surface distribution according to Khokhlova et al. 2000) and the observed one (Adelman \& Pyper 1985, Adelman 1997b) in different colours. Right: Comparison of the predicted light variations of HR 7224 (calculated using the iron and silicon surface distribution according to Lehmann et al. 2000) and the observed one (Adelman 1997a) in different colours.
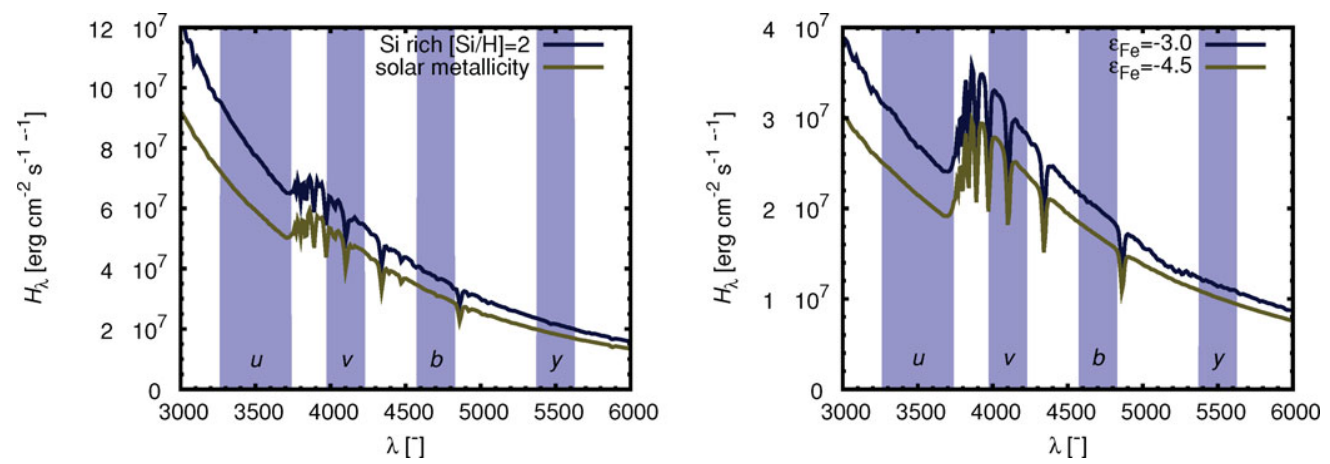

Figure 3. Left: Calculated fluxes from HD 37776 atmosphere with different silicon abundances. Theoretical flux was smoothed to better demonstrate the influence of silicon abundance. Silicon rich spots are brighter in uvby colours than the regions with low silicon abundance. Right: Calculated radiative fluxes from HR 7224 atmosphere with different iron abundances. Theoretical flux was smoothed to better demonstrate the influence of iron abundance. Iron rich spots are evidently brighter in uvby colors than the regions with low iron abundance.

Hubeny, I. \& Lanz, T. 1995, ApJ, 439, 875

Khokhlova, V. L., Vasilchenko, D. V., Stepanov, V. V., \& Romanyuk, I. I. 2000, AstL, 26, 177 Krtička, J., Mikulášek, Z., Zverko, J., \& Žižňovský, J. 2007, A\&A, 470, 1089

Krtička, J., Mikulášek, Henry, G. W., Zverko, J., Žižňovský, J., Skalický, J., \& Zvěřina, P. 2009, $A \mathscr{E} A, 499,567$

Lanz, T. \& Hubeny, I. 2007, ApJS, 169, 83

Lehmann, H., Tkachenko, A., Fraga, L., Tsymbal, V., \& Mkrtichian, D. E. 2007, A\&A, 471, 941

Mikulášek, Z., Krtička, J., Henry, G. W., Zverko, J., et al. 2008, A\&AA, 485, 585

Seaton, M. J., Zeippen, C. J., Tully, J. A., et al. 1992, Rev. Mexicana AyA, 23, 19 